\title{
Advance techniques in traumatic reticuloperitonitis diagnosis: review
}

\author{
Faisal G. Habasha Shaimaa N. Yassein \\ Coll. of Vet. Med./ Univ. of Baghdad. \\ email: faisalhabasha@yahoo.com
}

(Received 22 May 2013, Accepted 18 November 2013)

\begin{abstract}
Traumatic reticuloperitonitis (TRP) is a sporadic disease of ruminants that is caused by perforation of the reticulum by ingested foreign materials; it is a common reason for abdominal surgery in cattle. The disease may appear in forms of local and acute peritonitis, local chronic peritonitis and as unspecific form Anorexia, decrease in milk production, fever, ruminal stasis or ruminal hypo motility, tachypnea, grinding of teeth, reluctance to move and stance with an arched back and abducted elbows. The diagnoses are based on clinical examination, hematological examination, and other techniques.
\end{abstract}

Key words: Traumatic reticuloperitonitis (TRP), diagnosis, circulating cardiac troponin-I (cTnI) and cardiac troponin-T (cTn-T).

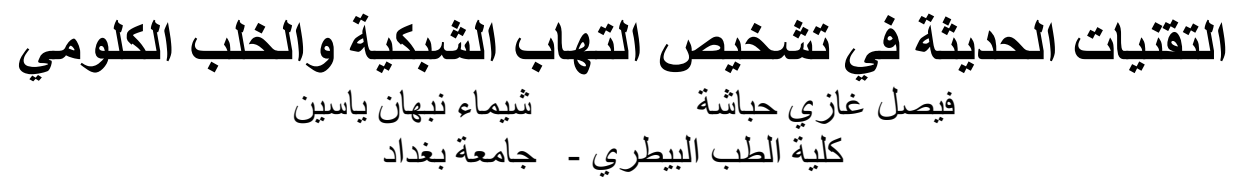

الخلاصة

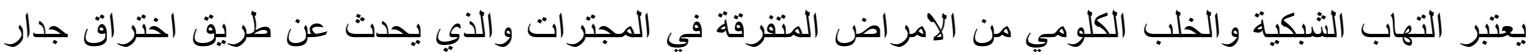

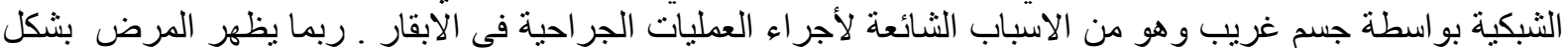

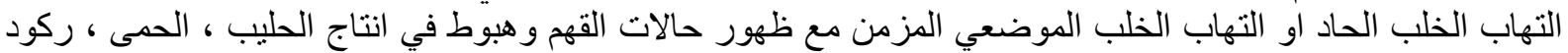

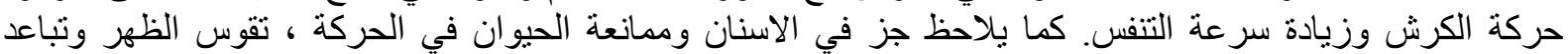

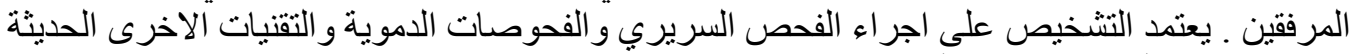

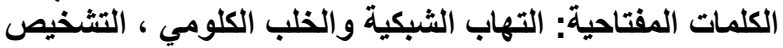

\section{Introduction}

Traumatic reticuloperitonitis (TRP) or hardware disease is a common disease of cattle but is rarely seen in small ruminants (1). It is the most common cause of anterior abdominal pain in cattle (2). The ingestive behaviour of cattle predisposes them to the accidental swallowing of thin sharp piece of metal foreign objects that settle in the reticulum (3). Ingestion of a foreign body may also be associated with diseases that cause pica, such as phosphorus deficiency. Subsequently, the foreign object may enter the reticulum and (a) without clinical diseases; (b) penetrate the reticulum wall only with intra-mural inflammation; (c) perforate the reticulum wall penetrate into the peritoneal cavity, and create localized peritonitis; or (d) migrate into the peritoneal and thoracic cavities. The diaphragm, pericardium, and heart muscle are located just cranial to the reticulum (4), with the liver positioned medially and dorsally and the spleen laterally and dorsally. These organs may sometimes be penetrated by foreign bodies and become involved in the inflammatory process. The importance of this disease is not only due to its higher prevalence among other digestive disorders, but also due to the difficulty in early prediction and difficulty in evaluation of its sequel by physical examination $(5,6)$. Therefore, an additional new diagnostic technique like ultrasonography is often helpful (7). It has been used for demonstration of physiological and pathological states of reticulum in cattle with 
TRP(8,9). Radiological examination of reticulum with the animal in dorsal recombency, is an accurate diagnostic method for the evaluation of cattle with suspected traumatic reticuloperitonitis (10). In spite of early diagnostic and prophylactic methods such as magnet implantation, metal detector and remover, there are still huge economic losses to be treated in the cattle industry $(11,12)$.

\section{Clinical signs}

The signs of TRP are dependent upon the site of reticular perforation and lesions caused by the foreign body in the surrounding areas. There are numerous scientific papers describing the clinical signs of TRP in cattle. The disease may appear in forms of local and acute peritonitis, local chronic peritonitis and as unspecific form (13); Anorexia, decrease in milk production, fever, ruminal stasis or ruminal hypo motility, tachypnea, grinding of teeth, reluctance to move and stance with an arched back and abducted elbows, signs of a heart rate of more than $100 \mathrm{bpm}$, distended jugular veins and muffled heart sounds or abnormal pericardial sounds. The heart rate was higher than normal, it ranged from 100 to $130 \mathrm{bpm}$. There are pericardial sounds, such as splashing, rubbing or squeaking sounds. Both jugular veins are distended, oedema of the throat region, brisket and ventral abdomen are the most common signs $(14,15)$, as seen in Fig. (1).

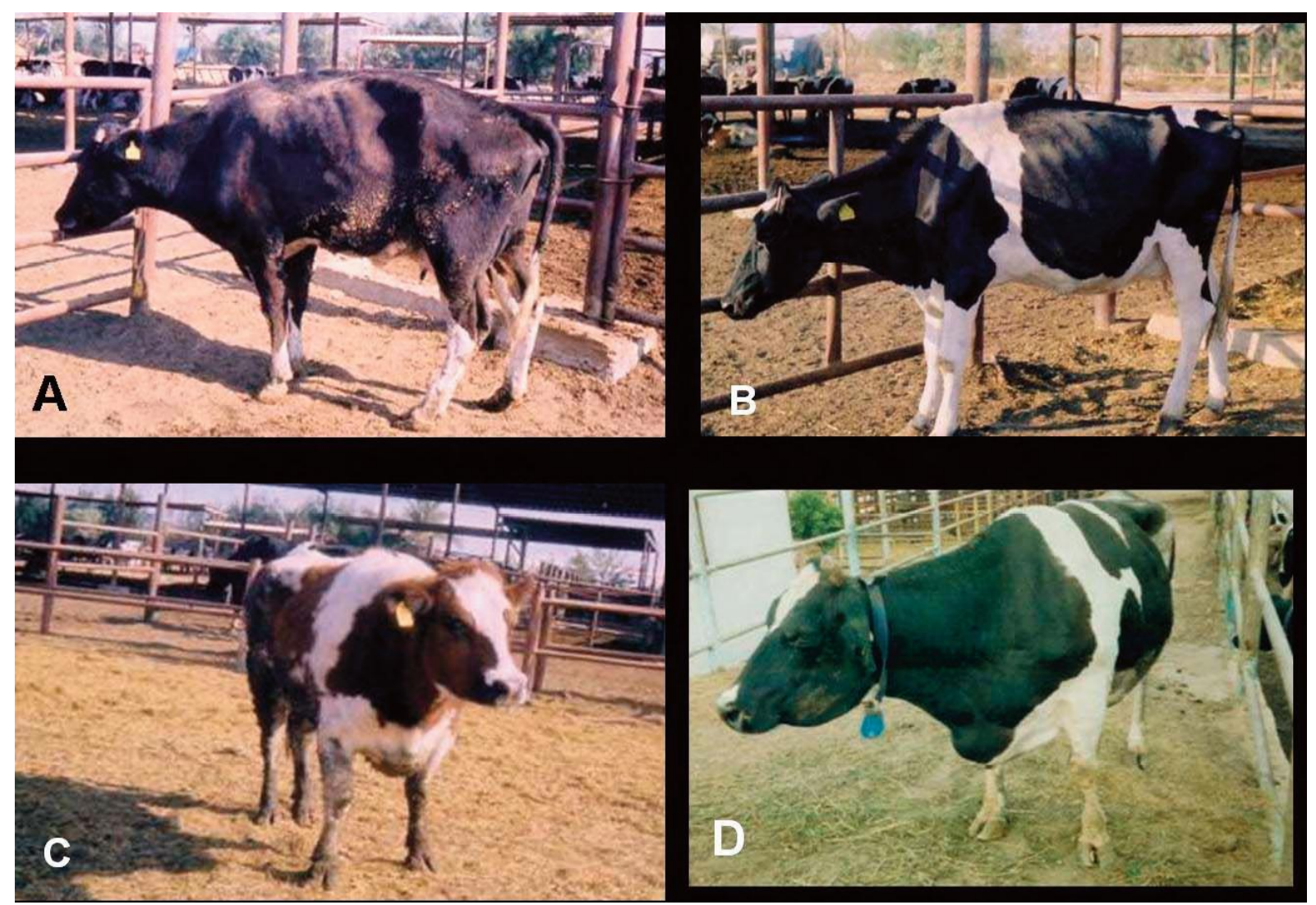

Fig. (1) Cows with TRP had arched backs (A), reluctance to move (B), and abduction of the forelimbs (C). Cows with TP had severe dullness and depression, and oedema of the brisket and submandibular regions (D).

\section{Diagnosis}

The complexity of development and the possibility that a number of syndromes can occur together makes the tentative diagnosis difficult. The disease must be distinguished from ketosis, simple indigestion and subclinical acute ruminal impaction (17). In practice, the diagnosis is mainly made by physical examination although additional diagnostic methods (such as hematologic tests, abdominocentesis, radiography, 
ultrasonography, laparoscopy or exploratory laparoruminotomy) might be used. Because complications of the disease are frequent, a more-accurate indication of its presence or severity would be valuable (18).

\section{Haematological examination}

There is significant erythrocytopenia (reduced RBCs) and lower $\mathrm{Hb}$ concentrations in the cows with TRP. On the other hand, PCV is significantly high in the cows with TRP. Moreover, there is significant leukocytosis, and lymphopenia in these cows $(19,20)$.

\section{In acute local peritonitis}

A neutrophilia, (mature neutrophils) and a left shift (immature neutrophils) are common. This is a regenerative left shift. Both the neutrophilia and the left shift will be increased on the first day and will last for up to 3 days, and in uncomplicated cases the count begins to return to normal. In chronic cases the levels do not return completely to normal for several days or longer periods and there is usually a moderate leukocytosis, neutrophilia and a monocytosis.

\section{In acute diffuse peritonitis}

A leukopenia with a greater absolute number of immature neutrophils than mature neutrophils (degenerative left shift) occurs, which suggests an unfavourable prognosis if severe. The degree of lymphopenia is an indication of a stress reaction to inflammation.(21). There is prolonged prothrombin time (PT), thrombin time (TT) and activated partial thromboplastin time (APTT). So, the TRP causes significant coagulation abnormalities and haematological alterations in dairy cattle (22).

\section{Biochemical analysis}

Biochemical analysis included spectrophotometric determination of serum glucose level, serum total protein and fibrinogen level, serum potassium and sodium level, serum chloride, serum urea nitrogen, serum creatinine, serum calcium, serum phosphorus, AST and ALP, serum lactate dehydrogenase $(\mathrm{LDH})$, and serum creatinine phosphokinase (CPK). There is an increase in total plasma protein (TPP) and plasma fibrinogen(PF) levels among cattle with TRP(23). Polyclonal gammopathy is characteristic for the electrophoretogram of cows with chronic local TRP (CL-TRP), and purulent pericarditis (PPC). Various degrees of hypoalbuminaemia, hyper-alphaglobulinaemia and low $\mathrm{A} / \mathrm{G}$ ratios are the associated changes in all cases of TRP. Hyper-beta-globulinaemia is noticed in cases with CL-TRP. Hyper-gamma-globulinaemia is evident in cases with acute local TRP (ALTRP), CL-TRP. Hyperproteinaemia is noticed in cows with local complications of TRP including AL-TRP, CL-TRP and RA. Hypoproteinaemia associated with severe hypoalbuminaemia and very low $\mathrm{A} / \mathrm{G}$ ratios $(<0.4)$ characterized cows with acute diffuse TRP(AD-TRP), PPC or fibrinous pericarditis (FPC). So, the concentrations and electrophoretic patterns of serum proteins in cow differ according to the anatomical location of the foreign body and the associated pathological lesions.(24). Sodium, potassium, and chloride levels are significantly lower in the cows with TRP than in the normal; however, AST, ALT, CPK, $\mathrm{LDH}$, blood urea nitrogen, and creatinine are significantly higher in the TRP than in the control group and the glucose level is significantly lower. The increase in liver enzymatic activity suggests that TRP is associated with impaired hepatic function that might be due to hepatic damage secondary to TRP(25). Circulating cardiac troponin-I (cTn-I) and cardiac troponin-T (cTn-T) can be used to determine myocardial cell damage in cattle with traumatic reticuloperitonitis (26). Troponin is a globular protein complex localised on thin filaments of striated muscle and consists of three subunits; Tn-T, Tn-I and $\mathrm{Tn}-\mathrm{C}$; all three subunits are integrally involved in the contraction and relaxation of the myofibrils (27). Troponin complex proteins have been determined in the heart muscle and blood of domestic animals (28). A previous study suggested that circulating cTn might be used for the diagnosis of cattle suffering from myocardial degeneration due to foot-and mouth disease (29), but the potential relationship between the acute myocardial cell damage caused by TRP and circulating $\mathrm{cTn}$ has not been studied extensively. Although serum cTnI concentrations were significantly higher in 
cattle with pericarditis compared with healthy cattle, they were not significantly different from concentrations in cattle with endocarditis, congenital cardiac disease, mediastinal abscess, reticulitis, caudal vena cava thrombosis, or chronic supurative pneumonia. Serum cTnI cannot be used to distinguish cattle with pericarditis from cattle with other primary cardiac diseases. In addition, serum cTnI concentrations cannot distinguish between cattle with primary cardiac diseases and those with other noncardiac, intrathoracic disorders(30).

\section{Qualitative cTn analyses:}

The concentration of cTn-T in heparinised blood is determined qualitatively by means of a commercial kit (Tropt Sensitive Rapid Assay; Roche) with an absolute detection limit of $0.08 \mathrm{ng} / \mathrm{ml}$. The concentration of cTn-I is determined in samples of serum with a commercial kit (card-I-kit Combo Test; AboaTech) with an absolute detection limit of $0.3 \mathrm{ng} / \mathrm{ml}$. Both tests were carried out according to the manufacturers' instructions and the results were recorded as positive when two purple lines appeared within 15 to 20 minutes of testing (Fig. 2) (26).

\section{Quantitative cTn-I analyses:}

A commercially available ELISA kit (CARD-I-KIT ELISA Troponin I; Labmaster) is used to determine the concentration of cTn-I according to the manufacturer's instructions. The optical densities of the samples were compared with a standard curve prepared from standards containing 0 to $14 \mathrm{ng} / \mathrm{ml}$ of cTn-I derived from human hearts. Mammalian myocardium has a high reactivity in the cTnmmunoassay, and this reactivity is highly selective, being more than 1000 times greater than in skeletal muscle. There are structural homologies between human beings and some animals for amino acid sequences of cTn-T, and the amino acid sequences are also identical among animals. (31). However, the critical question is whether high-sensitive troponin assays are clinically useful and in particular, whether some specific laboratory biomarkers (such as cTnI and cTnT) yield better diagnostic (or prognostic) accuracy and cost-effectiveness when compared with

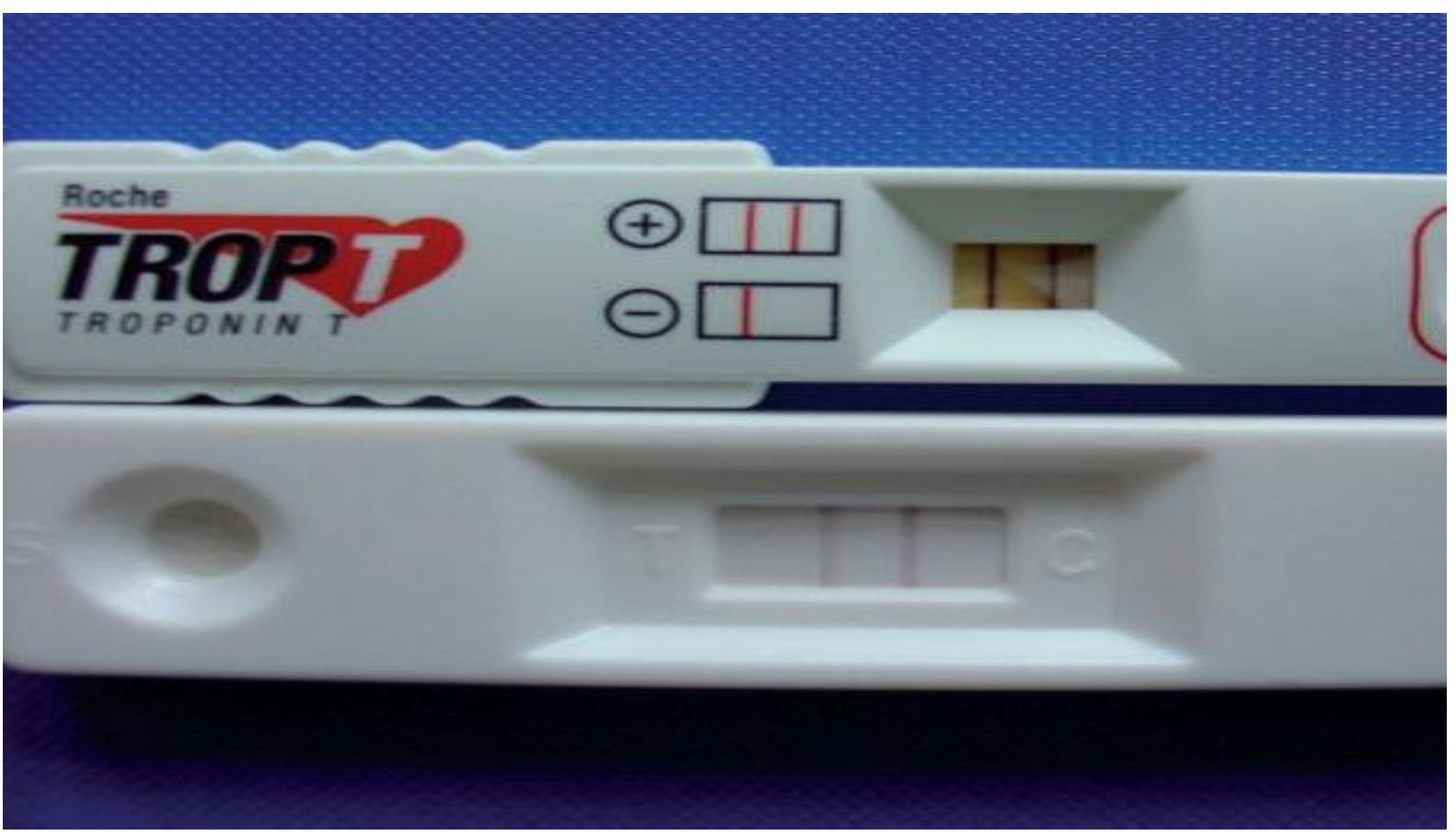

Fig. (2) Cardiac troponin-T (top) and cardiac troponin-I (bottom) test kits applied to cattle with traumatic reticuloperitonitis; two coloured lines appear in the reading windows if the result is positive, only one line appears if the result is negative. 
echocardiography in patients with cardiovascular disease. Only specific and well-designed clinical trials will answer this important question(32). Standardization of cardiac troponin I (cTnI) measurement is important because of the central role for diagnosis of myocardial infarction. In blood, cTnI is present as a heterogeneous mixture of different molecular species. The analytical problem caused by this heterogeneity may be circumvented by recognition of a unique, invariant part of the molecule that is common to all components of the mixture. Antibodies used for the development of cTnI assays should selectively recognize epitopes within this invariant part, leading to a consequential increase in the homogeneity of immunoassay reactivity. This should be associated with the use of a reference material that represents the natural and major antigen in blood after tissue release, i.e., the troponin complex. Although a primary reference material for cTnI is available, studies indicate that cTnI assays remain without harmony after recalibration using this material. To achieve closer comparability of cTnI values between assays, the use of a secondary reference material, consisting of a panel of human serum pools, is proposed for use by manufacturers to calibrate their assays. To assign true cTnI concentration values to this secondary reference material, establishment of a reference measurement procedure for cTnI is required.(33). The cTn-I concentrations ranged from 0.39 to 7.74 $\mathrm{ng} / \mathrm{ml}$ in the TRP cases in which the qualitative cTn-I kit was positive. Haptoglobin (Hp) is an a 2-globulin, synthesized in the liver. It is one of acute phase proteins whose serum levels increase in acute infections. It is believed that in cattle, $\mathrm{Hp}$ is involved in the regulation of lipid metabolism (34).The other form of Acute phase proteins (APPs)that is considered with the haptoglobin $(\mathrm{Hp})$ as diagnostic and prognostic biomarkers is serum amyloid A (SAA), which play an important role in the differential diagnosis of TRP using receiver operating characteristic (ROC) analysis.(35).Also, Total oxidant and antioxidant capacities and nitric oxide levels are important in cattle with traumatic reticuloperitonitis (36).

\section{Laparoscopy}

Right flank laparoscopy using a flexible fiberoptic laparoscope, $14 \mathrm{~mm}$ diameter and $1120 \mathrm{~mm}$ working length, is a reliable diagnostic aid for the presence of traumatic reticuloperitonitis (37). Laparoscopy in cattle is a promising tool for clinical diagnosis and treatment. The lower cost of the materials available in addition to the possibility of an intervention on an animal that is sedated does not entail more costs than an exploratory laparotomy. The application of this tool during abdominal explorations and biopsies allows the avoidance of invasive and often useless surgical interventions and even with the diagnosis and prognosis of certain conditions(38).

\section{Metal detection}

Metal detectors were used at one time to aid in the diagnosis of traumatic reticuloperitonitis. Ferrous metallic foreign bodies can be detected with metal detectors but the instruments are of limited use because most normal dairy cows are positive for metal over the reticular area.(21). Electronic metal detectors can identify metal in the reticulum but do not distinguish between perforating and nonperforating foreign bodies. The metal detector is an important auxiliary test, which has been used because it is a non-invasive, fast and cheap test. However, it does not determine if the foreign bodies are sharp or if they are really perforating the reticulum wall.(39).

\section{Radiography examination}

Radiography of the reticulum is very useful for the diagnosis of TRP. They are performed on standing animals and allow the detection of a metallic foreign body and the determination of its location in or outside the reticulum. Different parameters may be observed on radiographs for the diagnosis of TPR. They include presence or absence of a foreign body, position of the foreign body, presence of focal gas shadows or gas-fluid interface near the reticulum, the shape, size, and location of the reticulum. Of these parameters, location of the foreign body is 
the most reliable indicator for the diagnosis of TPR.(40).

\section{Ultrasonographic examination}

Abdominal ultrasonography is an excellent diagnostic and prognostic tool. It aids in deciding whether the animal should undergo surgical or medical treatment or be slaughtered (41). It is an ideal diagnostic tool for investigating gastrointestinal disorders in cattle. In animals with traumatic reticuloperitonitis, inflammatory fibrinous changes and abscesses can be imaged(42). So, ultrasonography provided exact information concerning the various sequel of TRP in animals. Moreover, ultrasonography made it possible to determine the location and extent of the lesions accurately, and the site best suited for abdominal and thoracocentesis. Reduced or absent biphasic reticular contractions and deposition of inflammatory materials on its serosal surface were the classical ultrasonographic identifies in TRP, according to (43-Mohamed and Oikawa,2007) described reticular and thoracic abscesses as circumscribed masses with an echoictoechogenic content. Healthy bovine reticulum appeared half-moon shaped with a smooth contour which plays a crucial role in the ruminant digestive tract because the primary cycle of rumen motility always starts with a reticular contraction (44). Ultrasonography is helpful for the diagnosis of traumatic reticuloperitonitis and differentiation of localised peritonitis from diffuse peritonitis $(45,46)$. Intraoperative echocardiography can be applied to evaluate the entire bovine pericardial sac and heart.(37).

\section{Confirmatory test}

Paracentesis (abdominocentesis and thoracocentesis) under ultrasonographic guidance, laparotomy and post-mortem examinations are used to confirm the diagnosis (47). Abdominocentesis and Pericadiocentesis may be performed blind or with ultrasound guidance (48). The best site for abdominocentesis is uncertain because the rumen occupies a large portion of the ventral abdominal wall and avoiding penetration of it is difficult. Cattle have a low volume of peritoneal fluid and failure to obtain a sample is not unusual (21). Laboratory evaluation of peritoneal fluid consists of determinations of total white blood cell count, differential cell count, total protein and culture for pathogens. The interpretation of the analysis of the peritoneal fluid can be unreliable because to date only a few correlations have been made between the laboratory findings and the presence or a absences of peritoneal lesions.

\section{PM examination}

In the TRP case sometimes there are extensive fibrinous adhesions between the cranioventral aspects of the reticulum, the ventral abdominal wall, and the Adhesions and multiple abscesses may be observed on either side of the reticulum. Large quantities of turbid, foul-smelling peritoneal fluid that contained fibrinous clots were present. The hearts in the TP group exhibited thickening of the pericardial sac. Cross sections of the pericardium and heart muscle showed thickening of the pericardium, with accumulation of pus between the pericardium and cardiac muscle (49).

\section{Histopathological examination}

Shows thickening of the pericardium due to accumulation of fibrinous inflammatory exudate, which was apparent between the pericardium and myocardium. With high magnification there was a fibrinous network observed to trap inflammatory cells: mostly neutrophils and mononuclear cells (50). The myocardiums in the TP cows have severe inflammatory cell infiltration replacing the cardiac muscle that has atrophied. Moreover, the myocardium of the cows severely affected by TP exhibits hyalinosis likewise, the myocardium of the cows with TP have severe inflammatory cell infiltration replacing cardiac muscle that has atrophied. Moreover, the myocardium of the cows severely affected by TP exhibited hyalinosis. These histopathological changes confirm the occurrence of pericarditis and myocarditis in the cows with TP, and support our echocardiographic findings. These changes are similar to those previously reported (17).

\section{In conclusion}

There is indicates that TRP and TP induced clinical, haematological, and 
biochemical changes in the affected cows. To date, this is the first comprehensive study to compare TRP and TP in cattle. TP induced more significant changes in the WBC (leukocytosis) and neutrophils (neutrophilia), and in the level of certain enzymes, such as liver (ALT and AST) and muscle (CPK and

\section{References}

1-Akkoc A (2007) Traumatic reticulopericarditis in a Saanen Goat. Turk J Vet Anim Sci; 31: 283-5.

2-Waldner CL, Kennedy RI, Rosengren L, Clark EG (2009) A field study of culling and mortality in beef cows from western Canada. Can Vet J.;50(5):491-9.

3-Braun U, Milicevic A, Forster E, Irmer M, Reichle S, Previtali M, Gautschi A, Steininger K, Thoma R, Zeller S, Lazzarini A, Manzoni C, Ohlerth S (2009) An unusual cause of traumatic reticulitis / reticuloperitonitis in a herd of Swiss dairy cows nearby an airport.Schweiz Arch Tierheilkd; 151(3):127-31.

4-Harwood D (2004) Alimentary tract perforation in cattle caused by tyre wire. Vet Rec; 154(18):574-5.

5-Smith BP (2009). Large animal Internal medicine.4th dition,MosbyUSA,894-850.

6- Maddy KT. Traumatic gastritis in sheep and goats. J Am Vet Med Assoc 1954; 124: 124-5.

7-Braun U, Goetz M, Marmier O (1993). Ultrasonography findings in cows with Traumatic reticuluperitonitis.Veterinary Record,133,416-422.

8-Floeck M. and Baumgart ner W. (2001). Ultrasonography diagnosis of Traumatic reticuluperitonitis and pericarditis in cattle (in German). Wiener Tieraerztliche Monatsschrift, 12, 347-354.

9-Floeck M.(2006). Ultrasonography of the reticulum -diagnostic tool for the practitioner.Solvenian Veterinary Research,43,208-209.

10-Farrow CS.(2006). Traumatic reticuluperitonitis. Vet. Clin. North Am. Food Anim. Pract. 15:397.

11-Ramprabhu R.,Dhanapalan P. and Prathaban S.(2003).Comparative efficacy of diagnosis tests in the diagnosis of Traumatic reticuluperitonitis and allied syndrum in cattle.Vet Med Assoc.,58,2-7.

12-Ramin AG.,Jafari Shoorijeh S.,Ahmadi Ashtiana MM.,Naderi MA.,Behzadi MA.and Tamadon A.(2008). Removal of metallic objects from animals feeds, Vet Scan,3, 1-7.

13-Hateley G (2004). Unusual Traumatic reticuluperitonitis in cow, Vet Rec ,154, 379-80.

14-.Abdelaal AM, Floeck M, Maghawry SLI and Baumgartner W (2009). Clinical and ultrasonographic differences between cattle and buffaloes with various sequelae of traumatic reticuloperitonitis. Veterinarni Medicina, 54, (9): 399-406.

15-Braun U, Lejeune B, Schweizer G, Puorger M, Ehrensperger F.(2007). Clinical findings in 28
LDH) enzymes, suggesting a more toxaemic and septicaemic reaction. The diagnosis of these cases was differentiated and confirmed by ultrasonographic examination of the reticular and cardiac areas. Moreover, the effects of TRP and TP could be confirmed by $\mathrm{PM}$ and histopathological examination.

cattle with traumatic pericarditis.Vet Rec. 20;161(16):558-63.

16-Mohamed T (2010) Clinicopathological and ultrasonographic findings in 40 water buffaloes (Bubalus bubalis) with traumatic pericarditis. Vet Rec. 20;167(21):819-24.

17-Steen A (2007) Field study on dairy cows with reduced appetite in early location: Clinical examination, blood and rumen fluid analyses. Acta Vet Scand.42,219-28.

18-Jafarzadeh,SR..; Nowrouzian,I.; Zohreh Khaki, Ghamsari,SM. and AdibhashemiF.(2004). The sensitivities and specificities of total plasma protein and plasma fibrinogen for the diagnosis of traumatic reticuloperitonitis in cattle. S.R. Jafarzadeh et al. Preventive Veterinary Medicine $65.1-7$.

19-Hakim A.,Mohindroo J., Kiranjeet S., Shwani K. andRandhawa CS.,(2010). Clinical, haematobiochemical, radiographic and ultrasonographic features of traumatic reticuloperitonitis in bovines.ATHAR Vol 80, No 7 .

20-Ocal, N., Gokce, G., Gucu, A.I., Uzlu, E., Yagci, B.B., Ural, K.:(2008). Pica as a predisposing factor for traumatic reticuloperitonitis in dairy cattle: serum mineral concentrations and haematological findings. J. Anim. Vet. Adv. 7: 651-656.

21-Radostis OM, Gay CC, Hinchcliff KW, Constable PD.( 2006) Veterinary Medicine: a textbook of the diseases of cattle, sheep, pigs, goats and horses. 10th ed. London: W.B Saunders,: 430-431.

22-Gokce HI, Gokce G, Cihan M.(2007). Alterations in coagulation profiles and biochemical and haematological parameters in cattle with traumatic reticuloperitonitis. Vet Res Commun. ;31(5):52937. Epub 2007 Jan 15.

23-Calloway, C.D., Tyler, J.W., Tessman, R.K., Hostetler, D., Holle, J., (2002). Comparison of refractometers and test endpoints in the measurement of serum protein concentration to assess passive transfer status in calves. J. Am. Vet. Med. Assoc. 221, 1605-1608.

24-Saleh M.A., Rateb H.Z., Misk N.A. (2008): Comparison of blood serum proteins in water buffaloes with traumatic reticuloperitonitis and sequelae. Research in Veterinary Science, 85, 208213.

25-Ocal, N., Gokce, G., Gucu, A.I., Uzlu, E., Yagci, B.B., Ural, K.:(2008). Pica as a predisposing factor for traumatic reticuloperitonitis in dairy 
cattle: serum mineral concentrations and haematological findings. J. Anim. Vet. Adv., 7: 651-656.

26-Gunes V, Atalan G, Citil M, Erdogan HM.(2008). Use of cardiac troponin kits for the qualitative determination of myocardial cell damage due to traumatic reticuloperitonitis in cattle. Vet Rec. 19;162(16):514-7.

27-Boccara, G., Pouzeratte, Y., Troncin, R., Onardet, A.,Boularan, A. M., Colson, P. \& Mann, C. (2000) The risk of cardiac injury during laparoscopic fundoplication: cardiac troponin I and ECG study. Acta Anaesthesiologica Scandinavica 44, 398-402.

28-Jesty, S. A., Sweeney, R. A., Dolente, B. A. \& Reef, B. A. (2005) Idiopathic and cardiac tamponade in two cows. Journal of the American Veterinary Medical Association 226, 1555-1558.

29- Gunes, V., Erdogan, H. M., Citil, M. \& Ozcan, K. (2005) Assay of cardiac troponins in the diagnosis of myocardial degeneration due to foot-and mouth disease in a calf. Veterinary Record 156, 714-715.

30-Mellanby R J, Henry J P, Cash R, Ricketts S W, Bexiga R, Truyers I, and Mellor D J (2009) Serum Cardiac Troponin I Concentrations in Cattle with Cardiac and Noncardiac Disorders. J. Vet. Internal Medicine.Vol.23:926-930.

31-Tate JR, Bunk DM, Christenson RH, Katrukha A, Noble JE, Porter RA, Schimmel H, Wang L, (2010). Standardisation of cardiac troponin I measurement: past and present. Pathology. ;42(5):402-8

32-Giannoni A, Giovannini S, Clerico A.(2009). Measurement of circulating concentrations of cardiac troponin I and $\mathrm{T}$ in healthy subjects: a tool for monitoring myocardial tissue renewal? Clin Chem Lab Med. 2009;47(10):1167-77.

33-Panteghini M, Bunk DM, Christenson RH, Katrukha A, Porter RA, Schimmel H, Wang L, Tate JR.(2008). Standardization of troponin I measurements: an update. Clin Chem Lab Med. 46(11):1501-6.

34-Nazifi S.; Rezakhani A.; Koohimoghadam M.; Ansari -lari M.; and Esmailnezhad Z..(2008). Evaluation of serum Haptoglobin in clinically health cattle and cattle with inflammatory diseases in Shiraz ,Atropical area in southern Iran.Bulgarian Journal of Veterinary Medicine, 11, No 2, 95-101.

35-Nazifi S, Ansari-Lari M, Asadi-Fardaqi J, Rezaei M.(2009). The use of receiver operating characteristic (ROC) analysis to assess the diagnostic value of serum amyloid A, haptoglobin and fibrinogen in traumatic reticuloperitonitis in cattle. Vet J.;182(2):315-319.

36-Atakisi E, Bozukluhan K, Atakisi O, Gokce HI.(2010). Total oxidant and antioxidant capacities and nitric oxide levels in cattle with traumatic reticuloperitonitis. Vet Rec. 4;167(23):908-909.
37-Bakos Z, Vörös K.(2011). Intraoperative echocardiography and surgical treatment of traumatic pericarditis in a pregnant cow.Acta Vet Hung. ;59(2):175-179.

38-Babkine M, Desrochers A.(2005). Laparoscopic surgery in adult cattle. Vet Clin North Am Food Anim Pract. 2005 Mar;21(1):251-79.

39-Mendes,L.C.N.,Feitosa,F.L.F., Haddad, F.N., Takada,L., Perri,S.H.V. and Peiro,J.R.(2009). Use of the Metal Detector to Determine the Prevalence of Metalic Foreign Bodies in Dairy Cows in Aracaluba Region, Barazil. Ars Vetrinaria Jaboticabal,SP ,v.25, n.2, 054-057,

40-Chaudhari SK.,Thorat MG., Yadav,GU., Mulani JB., Suryawanshi ,AA.and ngale,SS.(2009). Role of Radiological examination in diagnosis of Foreign body in bovines. Veterinary World, Vol.2(9):339.

41-Braun U.(2005). Ultrasound as a decision-making tool in abdominal surgery in cows. Vet Clin North Am Food Anim Pract. ;21(1):33-53.

42-Braun U.(2009). Ultrasonography of the gastrointestinal tract in cattle. Vet Clin North Am Food Anim Pract. ;25(3):567-90.

43-Mohamed T., Oikawa S. (2007): Ultrasonographic characteristics of abdominal and thoracic abscesses in cattle and buffaloes. Journal of Veterinary Medicine, Series A, 54, 512-517.

44-Braun U, Jacquat D.(2011). Ultrasonography of the reticulum in 30 healthy Saanen goats. Acta Vet Scand. 14;53:19.

45-Ghanem ,MM.(2010). A comparative study on traumatic reticuloperitonitis and traumatic pericarditis in Egyptian cattle Turk. J. Vet. Anim. Sci.34(2): 143-153.

46-Braun U, Lejeune B, Rauch S, Gorber U, Schweizer G.(2008). Ultrasonographic findings in 22 cattle with pericarditis traumatic. Schweiz Arch Tierheilkd. ;150(6):281-6.

47-Herzog K, Kaske M, Bischoff C, Kehler W, Hoeltershinken M, Starke A, Stöber M, Rehage J.(2004). Post-surgical development of inflammatory adhesions and reticular function in cows suffering from traumatic reticuloperitonitis. Dtsch Tierarztl Wochenschr. ;111(2):57-62.

48-Buczinski S, Boulay G, DesCôteaux L.(2011). Repeated pericardiocentesis as palliative treatment for tamponade associated with cardiac lymphoma in a Holstein cow. Can Vet J. ;52(6):663-6.

49-Ehsan T.; Mohammad R M.; Dezfoli1, Farhang S.; Farshad B.; Majid Sh.; and Majid .M.(2011). Traumatic reticulopericarditis (TRP) in sheep: Report of 4 cases in a herd. Slov Vet Res; 48 (2): 45-50.

50-Latimer, K.S., Mahaffey, E.A., Prasse, K.W.and Duncan(2003). Prasse's Veterinary Laboratory Medicine: Clinical Pathology, 4th ed., Ames, Iowa State Press, 68-77, 152-160. 